INPLASY

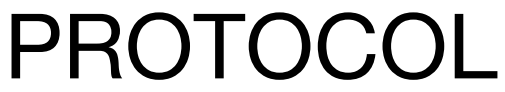

To cite: Garcia et al. Adjuvant effect of propolis to periodontal therapy for the treatment of periodontal disease: a systematic review. Inplasy protocol 202230030. doi:

10.37766/inplasy2022.3.0030

Received: 07 March 2022

Published: 07 March 2022

Corresponding author:

Martin Garcia

martin.garcia@upsjb.edu.pe

Author Affiliation:

Universidad Privada San Juan

Bautista.

Support: No.

Review Stage at time of this submission: Formal screening of search results against

eligibility criteria.

Conflicts of interest:

None declared.

\section{ADJUVANT EFFECT OF PROPOLIS TO PERIODONTAL THERAPY FOR THE TREATMENT OF PERIODONTAL DISEASE: A SYSTEMATIC REVIEW}

Garcia, $\mathrm{M}^{1}$; Tinedo, $\mathrm{P}^{2}$.

Review question / Objective: In patients with periodontal disease, what will be the scientific evidence on the adjuvant effect of Propolis to periodontal therapy for the treatment of periodontal disease?

Condition being studied: Periodontal Disease or Periodontitis, an inflammatory disease that affects the supporting tissues that surround the tooth, which are currently being studied with natural products that would work as an adjuvant to periodontal therapy and obtain better results.

Information sources: Three digital data sources were used, PUBMED, SCOPUS and EMBASE.

INPLASY registration number: This protocol was registered with the International Platform of Registered Systematic Review and Meta-Analysis Protocols (INPLASY) on 07 March 2022 and was last updated on 07 March 2022 (registration number INPLASY202230030).

\section{INTRODUCTION}

Review question / Objective: In patients with periodontal disease, what will be the scientific evidence on the adjuvant effect of Propolis to periodontal therapy for the treatment of periodontal disease?
Rationale: The bacterial imbalance within the oral cavity generates an increase in acidogenic and aciduric bacteria that communicate with each other through their cells, detecting quorum sensing, creating a solid and three-dimensional structure 
through a dynamic process, which is made up of exopolysaccharides, proteins and extracellular DNA. The formation of this acquired pellicle begins with a matrix of glycoproteins and salivary components that covers the surface of the teeth, which facilitates the adherence of primary colonizers such as gram-positive bacteria of the genus Streptococcus. The increase in this extracellular layer favors the aggregation of gram-negative bacteria such as Prevotella Intermedia, Fusubacterium or Capnocytophaga, responsible for the formation of a grayishyellow solid surface known as dental plaque, which exposes a considerable amount of chemical agents to the oral environment. that generate signs of inflammation in dental and periodontal structures, behaving as an etiological and pathological risk factor for Periodontal Disease. There are several effective ways to remove dental plaque, as well as the mechanical way, which is performed by the professional with prophylaxis or scaling and root planing, followed by daily brushing and antibacterial agents such as $0.12 \%$ Chlorhexidine, considered the Gold Standard, capable of controlling and eliminating bacterial plaque. However, the prolonged use of these chemical agents generates side effects such as taste alteration, surface staining, burning sensation, irritation and toxicity on oral cells. In addition, according to the World Health Organization (WHO), various microorganisms such as Staphylococcus Aureus have increased their resistance to antibiotics such as methicillin, becoming a challenging problem for the health professional. For this reason, studies have been carried out on the effects of natural products such as anise, lemon verbena, mint, oregano, among others, for their exploration as active biomolecules with antibacterial potential in periodontal disease.

Condition being studied: Periodontal Disease or Periodontitis, an inflammatory disease that affects the supporting tissues that surround the tooth, which are currently being studied with natural products that would work as an adjuvant to periodontal therapy and obtain better results.

\section{METHODS}

Search strategy: An electronic search was carried out in the following databases: PubMed, Embase and SCOPUS with the keywords: "Propolis", "Dentistry", "Periodontitis", "Periodontal disease", "Periodontal therapy".

Participant or population: Patients with Periodontal Disease.

Intervention: NON-SURG ICAL PERIODONTAL TREATMENT + PROPOLIS as a complement against Periodontal Disease.

C o m p a r a t or: NON-S UR G I C A L PERIODONTAL TREATMENT + PLACEBO as a complement against Periodontal Disease.

Study designs to be included: Randomized Clinical Trials.

Eligibility criteria: Articles published from 2014 to 2021 Scientific articles limited to the English languageArticles of studies randomized clinical trialsPresent specific quantitative methods for data collection.

Information sources: Three digital data sources were used, PUBMED, SCOPUS and EMBASE.

Main outcome(s): Clinical parameters such as Plaque Index, Gingival Index, Bleeding Index, Clinical Attachment Level and periodontal pocket depth were evaluated in each of the studies.

Quality assessment / Risk of bias analysis: The ROB2.0 tool will be used to assess Risk of Bias; The GRADE system will be used to assess the quality of evidence of results.

Strategy of data synthesis: The review of the quantitative and qualitative variables of the studies and the synthesis of the quantitative data will be carried out with 
the difference in averages for the variables Plaque Index, Gingival Index, Bleeding Index, Clinical Insertion Level, Periodontal Pocket Depth, performing a meta-analysis using the REVMAN 5.4.1 tool. Heterogeneity will be recorded with the inconsistency of 12 , in case of obtaining values greater than $50 \%$ it will be a value of heterogeneity of the included studies, performing a random effect statistical adjustment in the meta-analysis

Subgroup analysis: Not analyzed.

Sensitivity analysis: Not analyzed.

Language: No language restriction.

Country(ies) involved: Lima, Peru.

Keywords: Propolis, Periodontitis, Periodontal disease, Periodontal therapy.

Dissemination plans: Journal of International Society of Preventive and Community Dentistry.

Contributions of each author:

Author 1 - Martin Garcia.

Email: martin.garcia@upsjb.edu.pe

Author 2 - Pedro Tinedo.

Email: pedro.tinedo@upsjb.edu.pe 